\title{
The Psychology of Driving Automation: a discussion with Professor Don Norman
}

\author{
Neville A. Stanton, Mark S. Young \& Guy H. \\ Walker
}

School of Engineering \& Design, Brunel University, Uxbridge, Middlesex, UB8 3PH, UK

\begin{abstract}
Introducing automation into automobiles had inevitable consequences for the driver and driving. Systems that automate longitudinal and lateral vehicle control may reduce the workload of the driver. This raises questions of what the driver is able to do with this 'spare' attentional capacity. Research in our laboratory suggests that there is unlikely to be any spare capacity because the attentional resources are not 'fixed'. Rather, the resources are inextricably linked to task demand. This paper presents some of the arguments for considering the psychological aspects of the driver when designing automation into automobiles. The arguments are presented in a conversation format, based on discussions with Professor Don Norman. Extracts from relevant papers to support the arguments are presented.
\end{abstract}

Keywords: Automation, Adaptive Cruise Control, Mental workload, Mental under-load, Trust, Feedback

Reference to this paper should be made as follows: Stanton, N. A., Young, M. S. \& Walker, G. H. (200x) 'Human Factors in automobile automation: a discussion with Professor Don Norman’ Int. J. Vehicle Design, Vol. X, No. Y, pp.000-000.

Biographical notes: Professor Neville Stanton has degrees in Occupational Psychology (Hull, UK), Applied Psychology (Aston, UK) and Human Factors (Aston, UK). He holds a chair in Human Factors at Brunel University and conducts research in to areas as diverse as ground and air transportation, human supervisory control, command and control, team working, design methods and consumer products. Dr Mark Young has degrees in Psychology (Southampton) and Human Factors (Southampton). He has research interests in and air transportation, automation and workload. Dr Guy Walker has degrees in Psychology (Southampton) and Human Factors (Brunel). He has research interests in and air transportation, feedback, and command and control.

\section{Automation, Workload and Attention}

Every field has its luminaries. In Human Factors (as in every other domain) there are people who have produced that 'key' text or 'definitive work', people who propose ideas and concepts that lead one to ask, "why didn't I think of that?" In Human Factors research one such person is Professor Donald Norman. Many in the field of Vehicle Design may be familiar with this name, it is, after all, associated with a popular and 
widely read book, 'The Design of Everyday Things'(1990). Professor Norman has also written on the 'Problems of Automation' (1990), 'Emotional Design' (2003), 'The Invisible Computer' (1999) and held high profile positions at Apple Computers (Vice President of their Advanced Technology Unit). We have had the recent pleasure of exchanging ideas with him.

The discussion began with a request for copies of our papers on automobile automation. We sent some of the papers via email and post and a stimulating conversation ensued. So, rather than offering this as a 'scientific paper', the conversations are presented, interspersed with the relevant section from the papers, to help orientate the reader to several key arguments in Human Factors and its relation to vehicle design.

Research in our driving laboratory has largely been directed at understanding the effects of vehicle automation on driver performance, and more specifically, mental workload. The kinds of systems we have been looking at include Adaptive Cruise Control (ACC; a speed and headway keeping device) and Active Steering (AS; which keeps cars within their lanes). As many before us have stated, we found that some levels of vehicle automation can lead to underload - which is just as bad for performance as overload. But there remains controversy in the literature as to why underload is bad. We explain it with Malleable Attentional Resources Theory (MART).

EXTRACT FROM: Young, M. S. \& Stanton, N. A. (2002). Attention and automation: new perspectives on mental underload and performance. Theoretical Issues in Ergonomics Science, 3(2), 365-375.

Applied research on attention implicitly assumes that the size of resource pools is fixed. Capacity may change with long-term fluctuations in arousal, mood, or age (Kahneman 1973, Hasher and Zacks 1979, Humphreys and Revelle 1984), but in most applied experiments on attention these factors are assumed to be stable within participants. Performance on primary or secondary tasks, therefore, simply depends on demand not exceeding some arbitrary maximum. There is a possibility, though, that this limit may change in the relatively short term, depending on task circumstances. This introduces the concept of malleable attentional resource pools. Evidence is accumulating that simply reducing demand is not necessarily a key to improving performance. It is proposed that resources may actually shrink to accommodate any demand reduction, in a converse of the 'work expands to fill the time available' tenet. This could explain the apparent degradation of attention and performance observed in low demand tasks. If the maximum capacity of an operator has been limited as a consequence of the task, it is not surprising that they cannot cope when a critical situation arises. MART, therefore, potentially explains why mental underload can lead to performance degradation, whilst remaining grounded in established theories of attention.

Imagine someone driving a fully automated car. This is a situation which considerably reduces MWL. Assuming an attentional demand model of MWL (Liao and Moray 1993, Young and Stanton 2001), this translates to low demand on resources. Now, MART posits that the size of the relevant resource pool will temporarily diminish, as it is not required. This could result in poorer performance on any subsidiary tasks, or problems if the driver is suddenly faced with increased demand (e.g. if the automation fails).

The idea that the level of task demands can influence cognitive processing has been hinted at in previous research. Buck et al. (1994) quoted the 'par hypothesis' to explain some of their results. This states that, as demands fluctuate, operators increase or decrease the amount of effort invested in a task to maintain performance at a set level. 
This level represents an operator's personal par for that task. There is some support for this notion. Liao and Moray (1993) found that participants invest more effort with higher time pressure, which may increase capacity. Conversely, Desmond and Hoyes (1996) concluded that a decrease in performance at low levels of demand might be due to a failure to mobilize effort appropriately to match the task. MART reflects these attitudes, but is a little more parsimonious with respect to current knowledge. Being grounded in theories of attention, it does not have to appeal to extraneous concepts such as effort or motivation.

MART is also consistent with other theories of performance, such as working memory. An inverted-U relationship between arousal and performance could be due to competing processes of attentional resources and working memory. It has already been argued that MWL does not necessarily correlate with arousal directly, but the malleable resources perspective suggests that MWL can have the same influence, by affecting attentional capacity. In that respect, the hypothesis is by no means radical or novel, but simply taking existing ideas from the basic literature and applying them in a new domain.

A further implication concerns the traditional views of demand-performance relationships. Fixed capacity models assume that performance remains at ceiling, and is data-limited, as long as demands remain within the attentional capacity of the operator (Norman and Bobrow 1975, Stokes et al. 1990). Performance only begins to decline as the task demands approach the maximum resource availability. This is the very essence of the dual-task approach. Because two tasks can vary in objective difficulty, yet remain within the total capacity of the operator, overt performance differences will not be observed. A secondary task can assess remaining capacity once the primary task has taken its toll, and can, therefore, differentiate between such levels of difficulty. However, MART predicts that instead, performance is largely resource-limited for the full range of task demands. This would explain why some researchers (e.g. Roscoe 1992) have found an inverted-U relation between task demands and performance. At low levels of demand, attentional capacity is reduced, artificially limiting the performance ceiling. If task demands exceed the maximum capacity of the operator, performance degrades. Only at medium levels of demand are resources (and hence performance) optimized. These ideas are best understood in figures 2 and 3 [figure numbering from original paper]. 


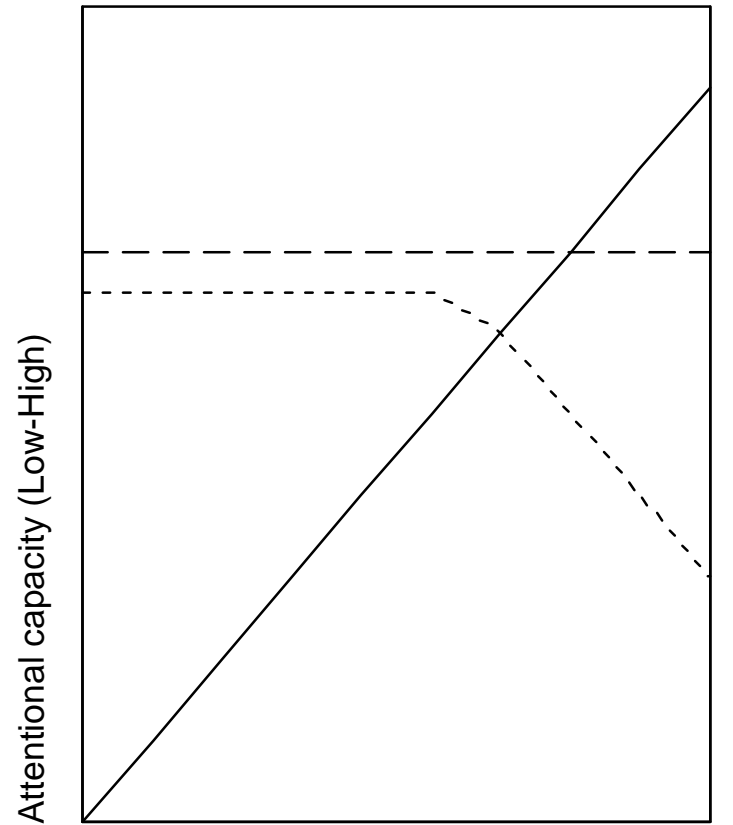

Task demands (Low-High)

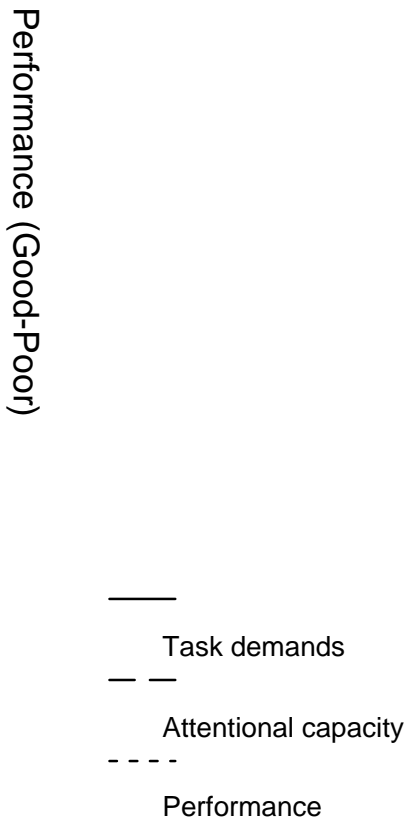

Performance

Figure 2: Relation between task demands and performance under a fixed capacity model (adapted from Stokes et al., 1990). Upper limit of attentional capacity is fixed (dashed line), and as task demands (solid line) approach that limit, performance (dotted line) degrades.

Figure 2 represents the textbook approach, in which performance remains constant until task demands begin to exceed capacity, reflecting the invariance of the capacity upper limit. However, in figure 3, the theory of malleable attentional resources has been applied to depress the upper capacity limit at lower task demands. This also limits the performance ceiling, effectively creating the classic inverted-U relation between task demands and performance. 


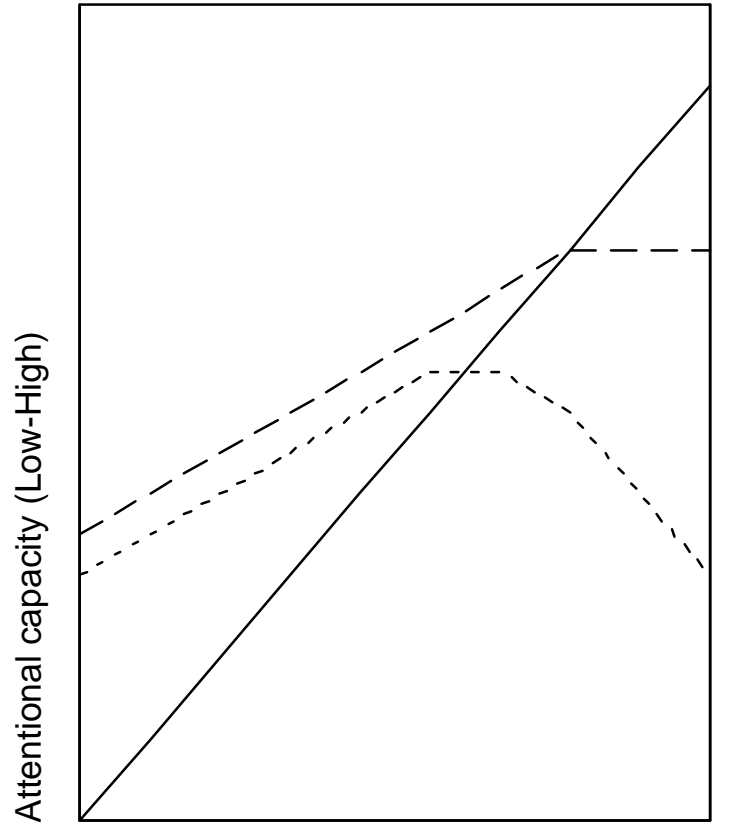

Task demands (Low-High)
0
$\frac{0}{7}$
0
3
0
0
0
0
0
0
0
$\frac{0}{1}$
0
0
0

$$
\begin{aligned}
& \text { Task demands } \\
& -- \\
& \text { Attentional capacity } \\
& \text { Performance }
\end{aligned}
$$

Figure 3: Relation between task demands and performance under a malleable attentional resources model.

Adopting a malleable attentional resources position would, therefore, help to explain the results from a number of studies in which performance and MWL are positively correlated (e.g. Roscoe 1992, Thornton et al. 1992, Scallen et al. 1995, Moss and Triggs 1997). Indeed, even basic memory research reviewed by Baddeley (1986) could be interpreted as support for MART. A positive correlation between memory span and concurrent reasoning was explained in terms of the demanding influence of errorcorrection, but the results are also consistent with a change in resource capacity.

On the basis of MART, it is predicted that excessively low mental workload, such as may be presented by automation, could result in a reduction of attentional resources. Young and Stanton (2002) used a neat measure of resource capacity to demonstrate that this was indeed the case. By comparing eye movements to responses to a secondary task, it was found that attentional capacity directly correlated with MWL. This was the first investigation into MART, and provided enough proof to warrant further investigations.

DON NORMAN: Underload: what a fascinating concept! I had not considered it before, but your analysis in the Human Factors paper is tantalizing. Now, couple that with Malleable Attentional Resources. I need time to digest this paper, as well as to read some of the papers you cite, and of course, the ones you are sending.

I obviously have some questions. For example, how well would your subjects respond to an unexpected emergency in the driving task (say, the need for sudden braking). 
If resources are truly diminished, then the braking response would be inferior for the situation with less attentional load. This would be a counter-intuitive result, which would greatly strengthen your hypothesis.

In this task, speed control was dramatically simplified for the manual condition because it is far easier to follow a car at a constant speed than to do so without the following behavior. Thus, it is possible that as far as speed control was concerned, manual and ACC were similar.

I haven't fully digested the implications of your attentional ratio measure. This seems to be hits divided by possible response time. Figure 1 shows hits increasing with the 4 conditions. Figure 2 [figure numbering from original paper] shows the ratio decreasing. So presumably time dramatically increases across the four conditions (well, I guess not much difference between manual and ACC). What does the ratio really mean, however?

'Figure 2': Attention ratio scores across each automation condition

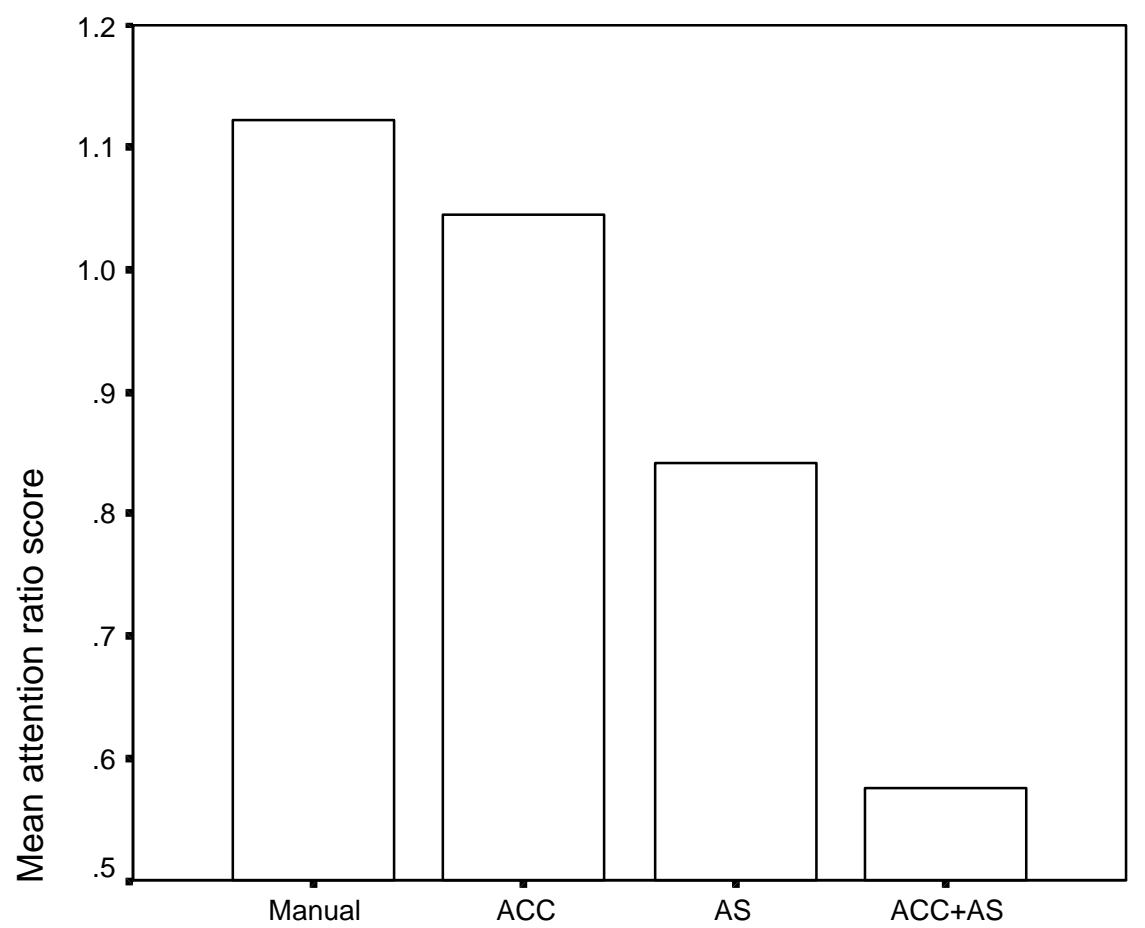

Anyway, I'm still thinking about this. But I believe underload to be a very important concept. MART probably is as well, but that requires more thought on my part - but it has passed the first test. I think this is fascinating work with some fascinating implications. I await the other papers.

\section{NEVILLE STANTON AND MARK YOUNG:}


The attention ratio was used to measure spare capacity, as it was expected that more time would allow more responses to the secondary task. We believe it essentially measures the effectiveness of a person's responses given the amount of time they're spending on it. For the sake of argument, let's say someone spending a minute on a task makes 60 correct responses. We could reasonably assume, then, that if they spent two minutes on the same task, they'd make 120 responses. However, in our underload conditions, that person might only be making, say, 80 or 100 responses. So although the driving task is easier, allowing them to spend more time on the secondary task, their responses aren't going up in proportion - and, indeed, are significantly fewer than they should be. It seems then, that proportionally fewer responses are accompanied with increasing levels of automation, which suggests to us that the attentional resource pool is somewhat diminished.

We have tried hard to rule out competing explanations (out-of-the-loop performance, as you note, as well as vigilance, mobilisation of effort, and trust in automation). The clincher comes, as you've foreseen, in an emergency scenario - I think that the emergency braking data would support the tenets of MART. We have done some exploratory studies of this sort and found that automation does not prepare the driver for emergencies; they do not use the spare capacity to engage in additional hazard detection and emergency response preparation activities. Quite the reverse appears to happen.

As predicted, participants in the underload conditions do not react as well as those who've been under higher workload. Interestingly, though, it's only the less skilled drivers who suffer, which I think is a result of automaticity, and actually supports MART even further. It's been said that emergency braking is an automatic response for skilled drivers, bypassing attentional resources - so although everyone suffers diminished attention, it doesn't matter so much for the experts. This has a whole bunch of interesting implications for automation design and driver training.

EXTRACT FROM: Young, M. S. \& Stanton, N. A. (2002). Malleable Attentional Resources Theory: A new explanation for the effects of mental underload on performance. Human Factors, 44 (3), 365-375.

The attention ratio score is possibly the single most important result to emerge from this experiment, and it provides the first piece of evidence in favor of malleable attentional resources theory. That participants' responses on the secondary task did not vary consistently with the amount of attention they directed to the task suggests that the size of the resource pool can change. On the basis of the malleable attentional resources hypothesis, it was expected that the attention ratio score would decrease in line with the MWL data from the secondary task. This prediction was directly upheld by the observed data, providing strong evidence for an association between task demands and attentional resource capacity.

These are encouraging results for MART. Further support is provided by the primary task performance data, as these reductions in demand are not accompanied by changes in driving performance. It could be argued that these results are attributable to different attention allocation strategies, or to a qualitative change in the driving task (from active operator to passive monitor), allowing more time to be devoted to the secondary task in the light of a perceived reduction in driving demands. If participants' allocation policies were inappropriate to the relative task demands, though, either a decrement in driving performance or an improvement in secondary task performance should be observed. This 
was not the case, driving performance remained constant regardless of attention ratio score, and no improvement in secondary task error rate was observed. Therefore, all attention devoted to the secondary task really did represent spare capacity. Furthermore, that driving performance did not improve with reductions in task demands implies that all spare capacity was allocated to the secondary task. It is reasonably safe to assume, then, that the sum of primary and secondary task demands reflected the total attentional capacity of the driver. Given this assumption, and the fact that increases in secondary task scores were not proportional to increases in visual attention, it is logical to conclude that attentional capacity had shrunk.

An alternative perspective might appeal to the classic vigilance decrement (e.g., Mackworth, 1948; Singleton, 1989) as an explanation for the results obtained here. However, the present experimental design does not qualify it as a vigilance task. Observations elsewhere (Singleton, 1989; Warm, Dember, \& Hancock, 1996) have typically found that a vigilance decrement sets in after 20 to $30 \mathrm{~min}$. Given the 10-min trials in the current study, it is unlikely that vigilance would have caused a problem. Furthermore, Parasuraman (1987) argued that continuous, dynamic tasks do not lend themselves to vigilance problems, and it is easily arguable that the task of driving fits these criteria. Therefore, malleable attentional resources theory seems to be a more likely explanation for these data.

Other alternatives relate to the nature of the task and center on issues of motivation and arousal. One might suggest that participants were simply bored or less motivated to maintain performance on the secondary task in the underload conditions. If this were the case, it would be expected that a lack of motivation would have a general effect on performance. Because performance on the primary (driving) task was not affected, the balance of evidence favors MART. Similarly, although physiological arousal was not measured in the present study, all of the experimental conditions posed fairly equal levels of physical demand. There was no reason to believe a priori that physiological arousal would vary, and the counterbalanced conditions should have mitigated any confounding effects of motivation or arousal. However, it is acknowledged that mental demands might have influenced attentional capacity only via an effect on arousal. Future research could strengthen the argument for MART by including measures of motivation and arousal.

From a theoretical point of view, the possible influence of arousal actually gives MART more support from the established literature. Kahneman's (1973) original model of attention explicitly identified physiological arousal as a factor in attentional capacity. If future investigations into MART cannot dissociate mental demands from arousal in determining resource size, then the contribution of this research will be limited to resurrecting ideas that have since been glossed over in the applied literature. In our opinion, such a contribution is no less valuable than validating the new theory itself. Indeed, there are practical advantages in the link with arousal because it is more readily detected than mental underload and may help with the design of monitoring systems.

In terms of the design implications, then, one might controversially conclude that drivers should be given extra tasks in their automated cars in order to maintain capacity at optimal levels. This denies all best practice in human factors, which prescribes a holistic approach to designing systems around the driver. Most ergonomists now agree that systems should be designed to support the driver rather than to replace them (Alm, Svidén, \& Waern, 1997; Grote, Weik, Wafler, \& Zolch, 1995; Stanton \& Young, 1998). The technology behind ACC could feasibly be used to present distance and/or relative speed information to the driver, rather than to actively control the vehicle itself. This 
solution would also satisfy the driver's need for feedback, a classic problem with automated systems (Norman, 1990).

DON NORMAN: All the people in the auto companies that I talk with defend the use of automation because it will "relax" the driver. Hmm. Your analysis is that it is not wise to relax the driver.

What I like about your approach is that it distinguishes underload from being "out of the loop": two very different concepts.

So, from your theoretical work, we should be able to compute an ideal state of attentional resource allocation -- or if you will, an ideal cognitive workload. Too small is bad. Too high is bad. Where is optimal?

NEVILLE STANTON AND MARK YOUNG: You are correct of course Don, we want an attentive driver rather than a relaxed one. We think that it should be possible to plot attention-workload-performance curves, but (as with all psychological phenomena) there will be big individual differences.

The theoretical implications do predict an inverse-U relation between workload and performance, as has long been suspected (see our TIES paper). However, the maximum of the curve is never going to be the same for everyone, nor under different situations. So we still have a fair way to go on defining the 'redline' of underload!

There seems to be little evidence that insights such as these have been incorporated into current (and, so far as we can tell, future) vehicle technologies. We have postulated that the driver could well find him or herself fighting with these systems, which would be reminiscent of the 'problem' with automation described in your paper. In it, you describe it as "not the presence of automation, but rather its inappropriate design. The problem is that the operations under normal operating conditions are performed appropriately, but there is inadequate feedback and interaction with the humans who must control the overall conduct of the task. When the situations exceed the capabilities of the automatic equipment, then the inadequate feedback leads to difficulties for the human controllers.” (Norman, 1990, p. 585). Although the paper gives several examples from the aviation industry, it appears to us that the words 'human' and 'task' in the quote above could easily be replaced with 'driver' and 'driving'.

EXTRACT FROM: Norman, D.A. 1990, The ' problem' with automation: inappropriate feedback and interaction, not 'over-automation'. Phil. Trans. R. Soc. Lond. B. 327, 585-593.

\section{a. We do not know enough to mimic natural human interaction}

Note that the problems in all three of the [aviation] case studies were not due to a lack of information, at least not in the technical sense. Autopilots work by physically moving the same controls that the pilots use. In the case studies of the loss of engine power and the fuel leak, the autopilots compensated by turning the control wheels. In theory, the crew could have noted the problem quite early by noting the position of the wheels, just as the second officer did note an abnormality in the fuel gauge readings in the fuel leak case study. Similarly, there was sufficient information in the case of pilot incapacitation. In these cases the problem was that no person or system commented upon the issues, so that 
nothing brought the potential problem to the attention of the relevant people. The feedback was potentially available, but it was not attended to properly. (During the writing of this paper, I took part in an informal replication of the fuel leak incident in the NASA-Ames full-vision, full-motion 727 simulator. Once again, the second officer failed to note the discrepant control wheel position, even though in this case he had read the relevant accident report: the normal cockpit activities drew the focus of attention away from the control wheel position. Our analyses afterwards indicated that the wheel position was not a very salient clue in any case. We plan further studies including a careful replication of this situation as well as a formal experimental study of the two 'thought experiments' described in this paper.) The task of presenting feedback in an appropriate way is not easy to do. Indeed, we do not yet know how to do it. We do have a good example of how not to inform people of possible difficulties: overuse of alarms. One of the problems of modern automation is the unintelligent use of alarms, each individual instrument having a single threshold condition that it uses to sound a buzzer or flash a message to the operator, warning of problems. The proliferation of these alarms and the general unreliability of these single-threshold events causes much difficulty (see Patterson 1989, Sorkin 1989, and Sorkin, Kantowitz, \& Kantowitz 1988). What is needed is continual feedback about the state of the system, in a normal natural way, much in the manner that human participants in a joint problem-solving activity will discuss the issues among themselves. This means designing systems that are informative, yet nonintrusive, so the interactions are done normally and continually, where the amount and form of feedback adapts to the interactive style of the participants and the nature of the problem. We do not yet know how to do this with automatic devices: current attempts tend to irritate as much as they inform, either failing to present enough information or presenting so much that it becomes an irritant: a nagging, 'back-seat driver', secondguessing all actions.

\section{b. A higher order of awareness is needed}

To give the appropriate kind of feedback requires a higher level of sophistication in automation than currently exists. Consider what is required for an automatic pilot to note that it is compensating more than normal. The current automatic systems are feedback loops that attempt to maintain a constant system state. To provide self-monitoring capability that would let it recognize that conditions are changing and more and more compensation is being used, would require a kind of higher-level of awareness, a monitoring of its own monitoring abilities. Now, obviously, it would not be difficult to build automatic systems for the specific cases of monitoring for increased rudder or control-yoke compensation, or for inappropriate fuel loss: any competent computerscientist could write an appropriate program. But what about the next problem, one that will involve yet a different system, yet a slightly different anomaly? We do not know how to solve the general condition. Consider what would be required of a fuel monitoring system to detect that the fuel level of tank $\mathrm{x}$ was dropping, but that fuel was only supposed to be fed from tank y. To solve this problem, in the general case, requires an intelligent system, one that understands the implications of the various control settings of the system. There probably has to be a knowledge base of the systems in the aircraft plus an internal representation for the items that would allow the system to reason about the potential cases. This is the sort of thing done today in laboratories of artificial intelligence and cognitive science, but we do not know how to solve this problem, for the general case. Moreover, even if the automatic monitoring equipment were to note the existence of a system trend or discrepancy that could lead to a difficulty later on, how should it be brought to the attention of the operators in a natural, intelligent fashion, much the way that normal cockpit conversation works? The solutions will require higher levels of automation, some forms of intelligence in the controls, an appreciation for the 
proper form of human communication that keeps people well informed, on top of the issues, but not annoyed and irritated. Our current level of knowledge is not enough to do these things.

\section{c. The new irony of over-automation}

Many ills have been laid at the feet of 'over-automation'. Too much automation takes the human out of the control loop, it deskills them, and it lowers morale. One much remarked-upon irony of automation is that it fails when it is most needed. I agree with all the analyses of the problems, but from these analyses, I reach the opposite conclusion, a different irony: our current problems with automation, problems that tend to be blamed on 'over-automation', are probably the result of just the opposite problem-the problem is not that the automation is too powerful, the problem is that it is not powerful enough.

\section{d. Why don't current systems provide feedback?}

Why do current systems have such poor feedback and interaction? In part, the reason is a lack of sensitivity on the part of the designer, but in part, it is for a perfectly natural reason: the automation itself doesn't need it! That is, if a designer is asked to design an automatic piece of equipment to control some function, the task is completed when the device functions as requested. Providing feedback and monitoring information to the human operators is of secondary importance, primarily because there does not appear to be any need for it. Feedback is essential because equipment does fail and because unexpected events do arise. In fact, in any complex task or environment, one should always expect unexpected events: what is unexpected is the type of event that will occur.

Human operators need to cope with these situations, and this is why the feedback and 'conversation' is required. Were the equipment never to fail, were it capable of handling all possible situations, then the human operator would not be necessary, so the feedback and interaction would similarly not be necessary. Today, in the absence of perfect automation an appropriate design should assume the existence of error, it should continually provide feedback, it should continually interact with operators in an appropriate manner, and it should have a design appropriate for the worst of situations. What is needed is a soft, compliant technology, not a rigid, formal one.

DON NORMAN: Just a short note to say how much I am enjoying reading your papers. I think you have done a really excellent job of bringing together all of the issues. But is anyone in the product side of automobiles listening?

I particularly liked your review paper. I realize this is five years old and that you have gone beyond this in your more recent work. Still, it's a great review and includes many other critical factors (e.g., trust).

But you have done a magnificent job of giving me material to ponder. So thank you.

NEVILLE STANTON AND MARK YOUNG: It is good to hear that you are enjoying our work, praise indeed! We have worked with vehicle manufacturers in the past, but mainly chasing technology rather than anticipating it. We were involved in an ACC project in the late 1990s and managed to get some recommendations incorporated into the first generation systems.

EXTRACT FROM: Stanton, N. A., \& Young, M. S. (2000). A proposed psychological model of driving automation. Theoretical Issues in Ergonomic Science, 1(4), 315-331. 
Whether we like it or not, automation is gradually taking over the driver's role. Full vehicle automation is predicted to be on British roads by 2030 (Walker, Stanton and Young, 2000). Whilst it is accepted that some drivers will still want to control their vehicles manually, many may be pleased to relinquish the role to automatic systems. Many of the computing technologies have been grounded in aviation systems (Billings, 1997; Stanton and Marsden, 1996), and technologies like adaptive cruise control are taking over from the driver already (Richardson, Barber, King, Hoare and Cooper, 1997). Adaptive Cruise Control (ACC) heralds a new generation of vehicle (Stanton, Young \& McCaulder, 1997). ACC controls both speed and headway of the vehicle, braking with limited authority in the presence of a slower lead vehicle, and returning to the set speed when the lead vehicle disappears (Richardson, Barber, King, Hoare and Cooper, 1997). In this way ACC differs from traditional Cruise Control (CC) systems. In traditional cruise control, the system relieves the driver of foot control of the accelerator only (i.e., relieving the driver of some physical workload), whereas ACC relieves the driver of some of the decision making elements of the task, such as deciding to brake or change lanes (i.e., relieving the driver of some mental workload), as well as physical demands of accelerator control. Potentially, then, ACC is a welcome additional vehicle system that will add comfort and convenience to the driver (Richardson et al., 1997). However, certain psychological issues do arise when considering any form of automation and these need to be properly addressed to improve overall system performance. It is envisaged that although the ACC system will behave in exactly the manner prescribed by the designers and programmers, this may lead to some scenarios in which the driver's perception of the situation is at odds with the system operation (Stanton and Young, 1998). Indeed, even those developing the systems recognise that "headway control raises the issue of whether the system matches the driver expectations with regard to braking and headway control” (Richardson et al., 1997; p. 91).

Most of the literature on driver behaviour tends to be restricted to the examination of a very limited set of variables. Whilst it might be argued by the researchers that this focus is necessary in order to determine the importance of the interaction between two or three important variables, it does not constitute the complexity of interplay between variables in the world at large. Sophisticated experimental design and analysis methodologies are able to cope with this complexity however, to provide an understanding of multiple interacting variables necessary to develop a psychological model of driving with automation. The purpose of this paper is, then, to identify the relevant the psychological variables and, on the basis of the literature, propose a psychological model.

An in-depth analysis of the psychological factors associated with the operation of automated systems is required to enable recommendations to be developed. The pertinent factors were elicited from a systems model of the driver-automation-vehicle as shown in figure 1, discussed by Stanton and Marsden (1996). From this figure, some potential psychological constructs emerge (see figure 2). 


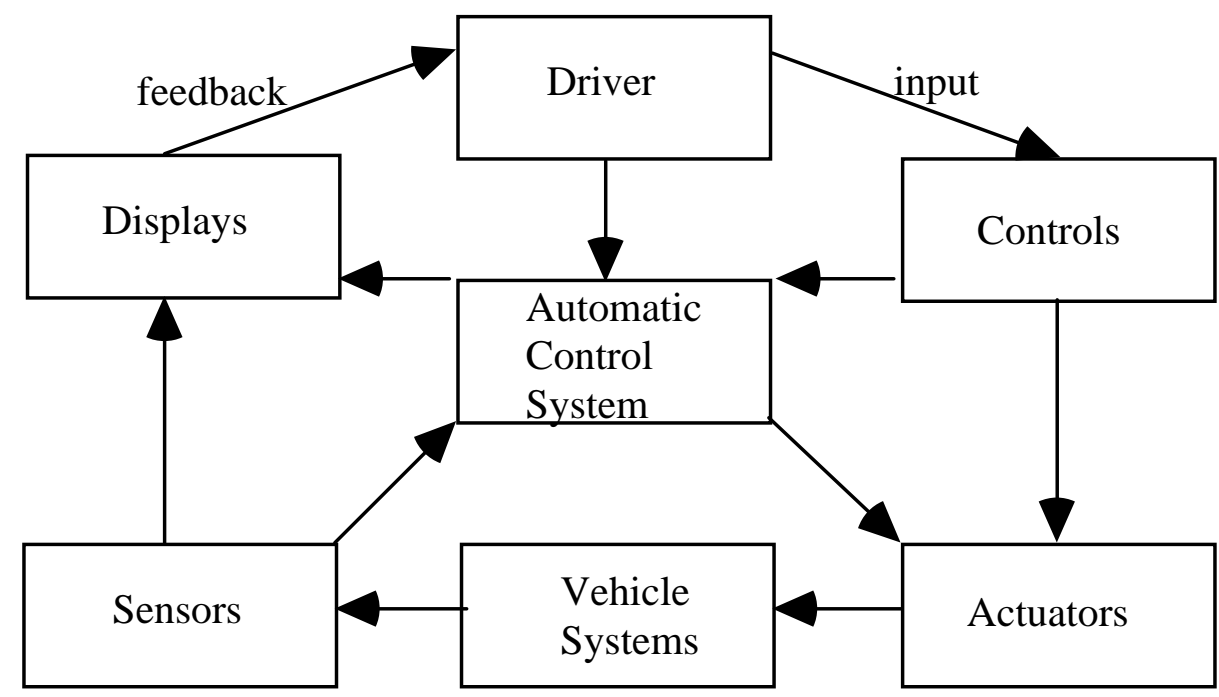

Figure 1. Information flow between driver, automatics and vehicle sub-systems (from Stanton and Marsden, 1996).

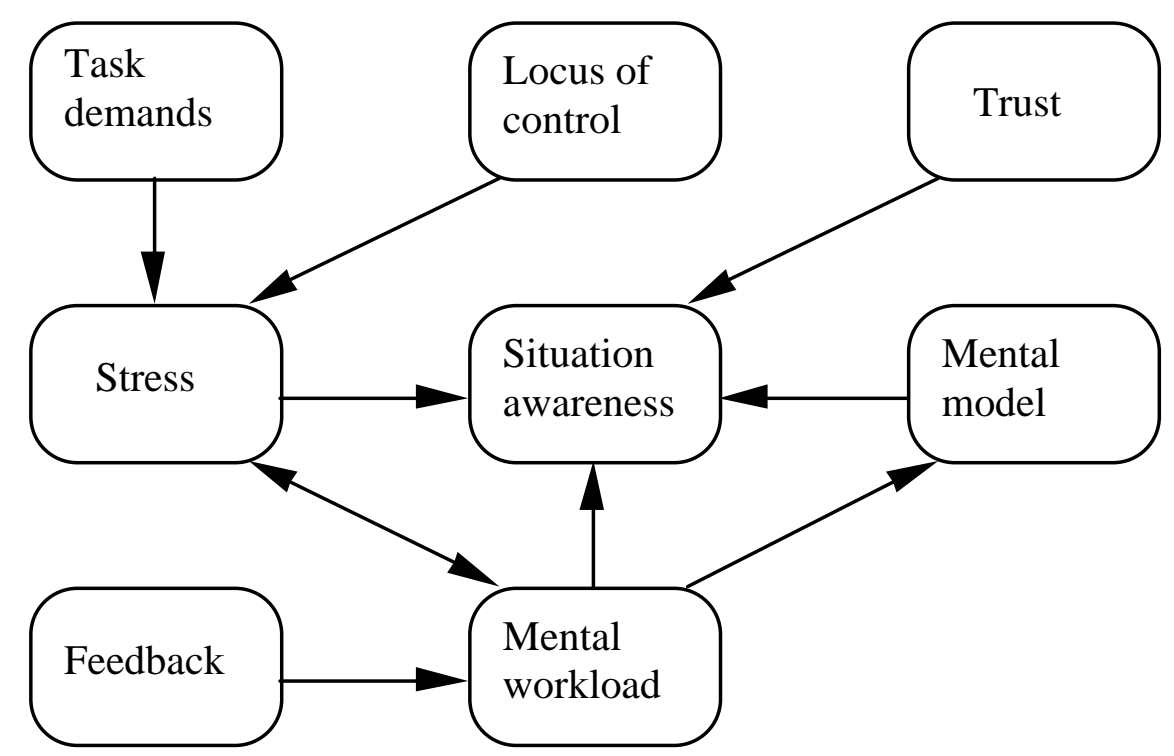

Figure 2. Hypothesised relationship between psychological factors

Most obvious is the issue of feedback, as can be seen as the information flows around the subsystems. Of particular interest is the role of feedback to the driver from the automated 
system. Typically, this tends to be poor (e.g., the lack of feedback from automated systems in the domain of aviation has been implicated in some incidents, Norman, 1990), because the automated systems do not require the feedback to function. Relatedly, the development of the driver's trust in the automated system may depend upon appropriate feedback. According to Muir and Moray (1996), the amount of feedback sought from an automated system by a human operator is directly related to the degree of trust they have in it to perform without failure. Passing control of the vehicle to a computer raises the issue of locus-of-control in the driver: does the driver feel that they, or the computer, is ultimately in control of the automobile? The degree to which a symbiotic relationship exists between the driver and the automatic system could determine how successful vehicle automation is perceived. One of the claims of all forms of automation is that the demands placed upon human operators will be reduced (Bainbridge, 1983). Therefore, the effects upon mental workload need to be considered. The workload literature suggests that there is an optimum level that leads to enhanced performance, demands greatly above or below this level could have a negative effect the driver (Young \& Stanton, 1997), such as increased levels of stress. Matthews and Desmond (1996) have found that driver stress is an important factor in the drivers like, or dislike, of driving and is linked to their experience of mental workload. One of the central concepts in driver automation seems to be the extent to which the driver is aware of the state of the automatic system, and the impact that has on the vehicle trajectory through the world. This concept is called situational awareness and has been the subject of research in the field of aviation (Endsley, 1995). Situational awareness depends, to a great extent, upon the development of an accurate model of the world, that enables information to be interpreted and predictions of future states made. Therefore, the role of mental models will also be considered. All of these factors are well established in the psychological literature but have yet to be fully explored with respect to vehicle automation.

DON NORMAN: Thanks, Neville, for the paper on ACC. The following incident was told to me recently by a friend (Jim Hollan of UCSD). What to you make of it?

Driving on the highway with ACC. Lots of traffic, so the vehicle is travelling slowly. The car now reaches its exit point, so the driver turns off the highway on to the exit lane. But the driver had forgotten that he was in ACC mode. The ACC, noting the absence of vehicles in front, rapidly accelerated to highway speeds, which is quite dangerous on the exit lane. The driver braked in time, slowing the car and disengaging ACC.

This is a classical example of mode error. As far as I can tell, you have not addressed issues of this sort. What do you think?

NEVILLE STANTON, GUY WALKER AND MARK YOUNG: It strikes us that these incidents (including that of your friend) are rather like the mode errors seen in other transport domains. For example, the two state warning device fitted into train cabs that alerts driver's to upcoming events (like signals or speed restrictions); the driver 'losing track' of what the warning refers to has been cited in several major accidents and incidents. Likewise, in the aviation sector, there are numerous instances of the autopilot being inadvertently and unknowingly configured for one course of action when another was desired. The key issue seems to be an uncoupling of system state from the user's perception of the system state. This is a fascinating, yet serious issue. In our technology trends paper we have tried to imagine both positive and negative consequences of vehicle automation and technology. 
EXTRACT FROM: Walker, G. H., Stanton, N.A., and Young, M.S. (2001). Where is computing driving cars? A technology trajectory of vehicle design. International Journal of Human Computer Interaction, 13, (2), 203-229.

It can be anticipated from the data gathered for this survey that embedded computing and mechanical intervention will have reached new heights by 2015. The vehicle will become a fully integrated unit with all its primary mechanical systems electronically linked, communicating with each other to accurately adapt to the driver and the road circumstances. In doing this, safety, efficiency and enjoyment can be maximised. Two thought experiments permit speculation on what the driving experience is likely to change into within the next 15 years, from two alternative view-points. Firstly, an optimistic forecast, contrasted with a pessimistic forecast.

\section{Thought Experiment 1 - An Optimistic 2015 Test Drive}

Pulling out of the driveway, the handling management system rapidly detects, via Gsensors and inputs from the active yaw system that the vehicle is travelling on challenging twisting roads. Algorithms, or even neural networks within the engine management and active yaw control systems deduce that the driver is also adopting a more sporting driving style. These systems will then signal to the steer by wire and the active damping system to endow the chassis with greater, more responsive 'turn-in' characteristics, at the expense of straight line stability, in order to help and encourage the driver to use the vehicle's considerable reserves of grip and roadholding. At the same time the engine management will revert to performance orientated ignition and valve timing maps and the soft instrument cluster will give extra dominance to the rev counter, and other engine parameters such as oil pressure and temperature, that are relevant to the type of driving currently in progress. This all combines to provide the sort of feedback and driver enjoyment that befits a sporty orientated car. In addition, the handling and engine management software has been tailored to the sales market that this particular car was sold into. Furthermore, at the heart of the software running on the vehicle's embedded computing are the vehicle parameters and characteristics that endow it with it's own distinctive, unmistakable 'brand character'.

Upon joining the motorway, however, the ACC will automatically activate, as will the traffic information and GPS navigation system. These help the driver select routes that maximise the efficiency with which the road network can be used. Behind the scenes, the engine management will revert to ignition and valve timing maps that optimise fuel efficiency and the soft instrument cluster will give prominence to the speedometer. Recognizing that sporty levels of feedback can be tiresome and, therefore, inappropriate for motorway cruising, the steer by wire and active damping will adjust the chassis for comfort and straight line stability.

The driver eventually joins a platoon of vehicles in the outside lane of the motorway, with all the vehicles in the convoy sensing each other via radar. The driver just has to steer the vehicle and good progress is being made, with minimal physical effort.

However, an older car in the middle lane has not noticed the vehicle in front of it slowing and cuts sharply into the path of our car.

The car's 360 degree sensors detect an imminent collision. Almost instantaneously the drive by wire sensors detect that the driver is not using the vehicle's abilities to the full, so the vehicle steps in to help by initiating the ABS and cutting the vehicle's audio and communication system to reduce any superfluous distractions. The car's systems detect that the driver is now responding with collision avoidance manoeuvres. The active yaw 
control comes into action to encourage the driver to initiate a manoeuvre at the edge of the vehicle's performance envelope. All the time information is flowing between vehicle systems throughout the car, via the OSEK architecture. The active yaw control and ABS are talking to the engine management so that the driven wheels are helping in collision avoidance. The smart airbags already know that there are two occupants on board, an individual in the driver's seat who weighs $90 \mathrm{Kg}$ and a passenger in the front seat weighing $60 \mathrm{Kg}$ and if required would deploy accordingly as would seat belt pretensioners via powerful 42volt actuators. Fortunately, the collision avoidance system, by helping the driver to fully exploit the vehicle's capabilities, has enabled the potential collision to be avoided. The driver can recommence driving and complete their journey unscathed.

\section{Thought Experiment 2 - A Pessimistic 2015 Test Drive}

Pulling out of the driveway the driver accelerates hard whilst piloting the car along twisting country lanes. The car is certainly going where the driver is intending it to, but the feedback is a little confusing for the driver. Even normal drivers are very sensitive to vehicle handling characteristics (Hoffman \& Joubert, 1968) and thus the whole driving experience feels rather sterile, as though the controls are remote from the devices actually under control. Also, the dashboard persists in altering its appearance and layout, which the driver finds distracting.

Accelerating onto the motorway the autonomous ACC system automatically activates when the driver would prefer to remain in manual control for a while as they settle down to a desirable cruise speed. The navigation system attempts to be helpful, but the driver knows exactly where they want to go. Unfortunately, the voice recognition system is not responding very well and the driver has difficulty in telling the device to shut down.

More out of frustration than a desire to make progress, the driver sets the cruise speed to $100 \mathrm{mph}$ and sets about picking their way through slower traffic. There is no longer much need to use the vehicle's mirrors prior to indicating as collision sensors warn the driver if there is a car alongside using a virtual rumble strip that shakes the steering wheel.

Five miles down the road the driver joins a platoon of ACC equipped cars cruising in convoy in the outside lane of the motorway. The lead car has set their cruise speed to around 90mph, which in terms of target risk is about the level at which most drivers seem willing to exceed the $70 \mathrm{mph}$ limit by. Car drivers have, therefore, adapted the technology to their needs and preferences in an unanticipated manner; one in which the Traffic Police strictly forbid, though struggle to enforce. It is also implicitly assumed by other drivers that the lead vehicle is anticipating the road conditions further ahead on their behalf and the following drivers, apart from steering the vehicle, tend to disengage somewhat with the driving task. Besides, in-vehicle devices such as MP3 players and incar internet provide plenty of other things to do during cruising.

However, while passing a string of older cars in the middle lane the driver notices that brake lights are coming on, and suddenly a vehicle swerves across immediately in front of our vehicle. At the instant before the driver was about to accelerate and steer into a small vacant gap in the middle lane, in order to allow the older vehicle to miss the front of our car, the ABS cuts in violently. The collision avoidance system, of course, can't see brake lights, neither can it see small adjacent gaps that the driver wishes to use as a form of 'escape route'. It just knows that a proximity/speed threshold has been exceeded and that the maximum occupant protection is afforded in the longitudinal plane. Whilst sustaining an unexpected $1.2 \mathrm{~g}$ of deceleration the driver still tries to steer left to allow the skidding car to pass in front, but the collision avoidance system has other ideas. It tries to 
maintain the car in a straight ahead position, whilst still braking hard. The steer-by-wire technology is, under these circumstances, given full authority to override driver inputs. The driver and passenger are suddenly recoiled back in their seats as the seat belt pre tensioners activate, the drivers view is then totally obscured as the airbags deploy and a violent collision occurs at the front of the vehicle. Both occupants are shaken but otherwise completely unharmed; secondary (in not primary) safety is now far advanced. Unfortunately, the other older vehicle and its occupants have not survived the collision with the newer car very well at all.

\section{Concluding Thoughts}

Thought experiment 1 demonstrates how technology and embedded computing optimises the interaction that the vehicle has with the road, and how it can potentially enhance the interaction between the vehicle and the driver. The assumption in this case is that the implementation of vehicle technology has benefited from human factors research to mitigate the effects of negative behaviour adaptation due to RHT, misuse of the technology by the driver and inappropriate feedback. Conversely, thought experiment 2 demonstrates an alternative scenario where the vehicle and its associated technology is not interacting optimally with the psychology of the driver, leading ultimately to frustration and misuse. Relevant to both scenarios is the issue of partial integration, whereby the newer, technology laden vehicle, is sharing the road with older, less sophisticated vehicles with a range of consequences, especially for safety and efficiency.

All vehicle technologies have consequences not only for the interaction that the vehicle has with the road, and other vehicles, but also for the interaction between the driver, their vehicle, and the in-vehicle technology. This paper echoes the message of others in the field of advanced vehicle technology, in stating that a wide range of important human factors issues surround the implementation and use of such technology (Michon, 1993; Owens, Helmers, \& Sivak, 1993; Parkes \& Franzen, 1993). Research concerning human machine interaction is a growing area that has wrought measurable improvements, but in comparison to the vehicles interaction with the road (an area that is well understood and rapidly becoming optimised) there is still much that remains to be learnt.

It is fortunate that, to-date, vehicles have evolved relatively well to the needs and abilities of drivers. It is certainly difficult to conceive of a similar device of such mechanical complexity and an environment of usage with similar dynamism and potential danger, being interacted with so effectively and by such a diverse population of users. The pace of technological change, however, does not afford a similar luxury in terms of a further 100 years of evolution towards the solutions of integrating advanced driver technology with the capabilities and limitations of drivers. Human Factors provides a means, if you will, of 'short circuiting' the evolutionary envelope; a means to scientifically understand and to predict the ways in which driver behaviour will adapt and, one hopes, be improved by new technology. More importantly, an opportunity arises to use Human Factors much earlier on in the design process to derive truly innovative technological solutions to improving safety, efficiency and enjoyment.

\section{References}

Norman, D. A. (1998). The Invisible Computer: Why good products can fail, the PC is so complex, and information appliances the answer. Cambridge, MA: MIT Press. 
Norman, D. (1990). The Design of Everyday Things. New York: DoubleDay

Norman, D.A. (1990). The 'problem' with automation: inappropriate feedback and interaction, not 'over-automation'. Phil. Trans. R. Soc. Lond. B. 327, 585-593.

Norman, D. A. (2004). Emotional Design: Why We Love (or Hate) Everyday Things. New York: Basic Books.

Stanton, N. A. \& Young, M. (2001) A proposed psychological model of driving automation. Theoretical Issues in Ergonomics Science 1 (4), 315-331.

Young, M. S. \& Stanton, N. A. (2002). Malleable Attentional Resources Theory: A new explanation for the effects of mental underload on performance. Human Factors, 44 (3), 365-375.

Young, M. S. \& Stanton, N. A. (2002) Attention and automation: new perspectives on mental underload and performance. Theoretical Issues in Ergonomics Science, 3 (2) 178194.

Walker, G. H., Stanton, N.A., and Young, M.S. (2001). Where is computing driving cars? A technology trajectory of vehicle design. International Journal of Human Computer Interaction, 13, (2), 203-229.

\section{Other Papers On Automobile Automation By The Ergonomics Research Group At Brunel University (in chronological order)}

Stanton, N. A. \& Marsden, P. (1996) From fly-by-wire to drive-by-wire: safety implications of automation in vehicles. Safety Science, 24, (1) 35-49.

Stanton, N. A. \& Marsden, P. (1997) Drive-by-wire systems: automation of the driver's tasks. Proc. Instn Mech Engrs 211 (D) 267-276.

Stanton, N. A., Young, M. \& McCaulder, B. (1997) Drive-by-wire: the case of mental workload and the ability of the driver to reclaim control. Safety Science. 27 (2-3) 149159.

Young, M. \& Stanton, N. A. (1997) Automobile automation. International Journal of Cognitive Ergonomics. 1 (4) 325-336.

Stanton, N. A. \& Young, M. (1998) Vehicle automation and driving performance. Ergonomics. 41 (7), 1014-1028.

Stanton, N. A. \& Pinto, M. (2000) Behavioural compensation by drivers of a simulator when using a vision enhancement system. Ergonomics 43, 1359-1370.

Stanton, N. A. \& Pinto, M. (2001) Will radar-based vision enhancement make driving safer? Proceedings of the Institution of Mechanical Engineers - Part D 215, 959-967. 
Stanton, N. A.; Young, M. S.; Walker, G. H.; Turner, H, \& Randle, S. (2001)

Automating the driver's control tasks. International Journal of Cognitive Ergonomics 5 (3), 221-236.

Young, M. \& Stanton, N. A. (2001) Out of control. New Scientist 172, 44-47.

Walker, G. H., Stanton, N. A., \& Young, M. S. (2001). An on-road investigation of vehicle feedback and its role in driver cognition: Implications for cognitive ergonomics. International Journal of Cognitive Ergonomics, 5, (4), 421-444.

Young, M. S. \& Stanton, N. A (2004) Taking the load off: investigations of how Adaptive Cruise Control affects mental workload. Ergonomics 47 (8), 1014-1035.

Stanton, N. A. \& Young, M. (2005) Driver behaviour with Adaptive Cruise Control. Ergonomics 48, $1294-1313$

Stanton, N. A. \& Young, M. (2006) Automatic Intelligent Cruise Control. Journal of Intelligent Systems 15, 357-388.

Young, M. S. \& Stanton, N. A (2006) Back to the future: a review of brake reaction times for manual and automated vehicles. Ergonomics (in press).

Walker, G. H., Stanton, N. A., \& Young, M. S. (2006). The ironies of vehicle feedback in car design. Ergonomics, 49(2), 161-179.

Walker, G. H., Stanton, N. A. \& Young, M. S. (2006). What the Insidious Trend in Embedded Computing in Vehicles can tell us about Vehicle Feedback: A Comparison of Driver Situation Awareness Measures. International Journal of Human Computer Studies (in press) 